\title{
Environmental Tax, Urban Minimal Wage and Welfare
}

\author{
Cheng-Te Lee ${ }^{1}$, Kuo-Hsing Kuo ${ }^{1} \&$ Chen Fang ${ }^{2}$ \\ ${ }^{1}$ Department of International Trade, Chinese Culture University, Taiwan \\ ${ }^{2}$ Department of International Trade, Takming University of Science and Technology, Taiwan \\ Correspondence: Cheng-Te Lee, Department of International Trade, Chinese Culture University, No. 55, \\ Hwa-Kang Road, Yang-Ming-Shan, Taipei City 11114, Taiwan. Tel: 886-2-2861-0511 ext. 35135. E-mail: \\ lzd4@faculty.pccu.edu.tw
}

Received: August 20, 2013

Accepted: October 16, $2013 \quad$ Online Published: November 26, 2013

doi:10.5539/ijef.v5n12p86

URL: http://dx.doi.org/10.5539/ijef.v5n12p86

\begin{abstract}
This paper constructs a specific-factor model of a closed HT economy to examine the reasons for the government of China imposing on environmental tax and increasing the minimum wage simultaneously. We find that the government policies will decrease the pollution and increase the urban unemployment rate. In addition, we also prove that the impact on the welfare level will depend on the degree of disutility of pollution. If the degree of disutility of pollution is large enough, then the policies of China government will raise the welfare level.
\end{abstract}

Keywords: environmental tax, urban minimal wage, welfare

\section{Introduction}

Many developing countries like China, India, Mexico, and Chile, have attempted to promote the "industrialization" in order to reduce domestic poverty. As those countries not only progress but also increase employment through industrialization, the environment also deteriorates due to industrial pollution. As a result, they must suffer from downgrade of the quality of life. Therefore, it is quite well known that the issue of the traditional job-environment tradeoff is important in the developing country (Note 1).

In general, the industrialization is considered more important than environmental protection in the developing countries. However, a prominent example is that the world's biggest carbon emitter, China, has set the targets not only to reduce the amount of carbon dioxide by $20 \%$ by 2020, compared with 2005 levels (The Economist, 2009) (Note 2) but also to cut emissions of nitrogen oxides by $10 \%$ by 2015 (The Economist, 2011). To hit these targets, the government of China adopts some environmental preservation policies, for example, imposing on carbon tax. In addition, in the corresponding period, we also observe that the minimum wage rises continuously in China. In particular, since 2010, the government of China has increased the minimum wage by $25 \%$ (Common Wealth, 2011) (Note 3). Namely, the observation that the government of China imposes on environmental tax and increases the minimum wage simultaneously is observed. Therefore, the main purpose of this paper is to hope to explain why China would do that.

In the traditional literature of development economics, Harris and Todaro (HT) (1970) have provided a standard model to capture the economic phenomenon of a developing country with urban unemployment. However, in the HT model, the implement of minimum wage policy is an important reason for the existence of urban unemployment. Many studies extend the basic HT model to investigate the impact of the environmental preservation policy on the urban unemployment and the national welfare, including Dean and Gangopadhyay (1997), Chao et al. (2000), Daitoh (2003; 2008; 2012), Beladi and Chao (2006), Rapanos (2007), Tsakiris et al. (2008) and Daitoh and Omote (2011) (Note 4). However, these existing studies argue that the effects of environmental protection on urban unemployment and welfare are inconclusive.

Intuitively, a rise in the minimum wage rate would lead to a labor-migration from rural sector to urban sector and then the urban unemployment rate rises. Therefore, the impact of the minimum wage rate on welfare is ambiguous (Note 5). As mentioned earlier, the effects of environmental protection on urban unemployment and welfare are also inconclusive. Thus, the question posed in this paper is: How do the increases in environmental tax and minimum wage affect urban unemployment and welfare of an economy. 
As a complement to the literature, this paper will construct a specific-factor model of a closed HT economy to examine why the government of China imposes on environmental tax and increases the minimum wage simultaneously. We find that when the government raises the environmental tax and the urban minimal wage rate simultaneously, the pollution would decrease and the urban unemployment rate would increase. In addition, we also prove that the impact on the welfare level will depend on the degree of disutility of pollution. If the degree of disutility of pollution is large enough, the policies of China government will raise the welfare level. Therefore, if China government's objective is to increase the welfare level, then it will do that.

The rest of this paper is organized as follows. Section 2 establishes a closed HT model. Section 3 considers the impact of the environmental policy on unemployment and pollution. The impact of the environmental policy on welfare is examined in Section 4. Section 5 provides concluding remarks.

\section{The Model}

Consider a specific-factor model of a closed HT economy comprising two sectors, said the rural and urban sectors. The rural sector hires labor $L_{x}$ and sector-specific land $T$ to produce the agricultural good $x$. Assume that there is perfect competition in the rural labor market and land market. The urban firm produces the manufactured good $y$ using labor $L_{m}$ and the "dirty input" $z$. Suppose that all the commodity markets are perfect competition and that all firms in each sector and all consumers in this economy are identical. The production functions which are assumed to be constant returns to scale technology in both sectors can be specified as follows:

$$
\begin{aligned}
& x=f\left(L_{x}, T\right), \\
& y=g\left(L_{m}, z\right) .
\end{aligned}
$$

In the rural sector, labor and land are fully utilized. In the urban sector, the institutionally minimal wage rate $w_{m}$ prevails. The factor of production $z$ will not only lower environmental quality but also reduce consumers' utility and hence it can be viewed as "pollution". For simplicity, we assume that the pollution market does not exist. At the same time, the government will impose an environmental tax $\tau$ on the manufacturing firm's use of $z$. (Note 6) Let the agricultural good $x$ be numéraire. Hence, the zero profit conditions can be expressed as follows:

$$
\begin{gathered}
C^{x}\left(w_{x}, \eta\right)=1 \\
C^{y}\left(w_{m}, \tau\right)=p
\end{gathered}
$$

where $C^{x}(\cdot)$ and $C^{y}(\cdot)$ denote the unit cost functions of good $x$ and $y$ respectively. The variables $w_{x}$ and $\eta$ represent the rural wage rate and the rental rate of land respectively. The variable $p$ is the relative price of good $y$.

Assume that $L_{u}$ represents the unemployment population in urban sector. Let $\mu=L_{u} / L_{m}$ and thereby the unemployment rate is $\mu /(1+\mu)$ in urban sector which implies that the larger the variable $\mu$ is, the larger the unemployment rate will be. The labor migration between rural and urban sectors is determined by the HT migration condition (3) which indicates that the rural wage rate equals the expected urban wage rate $w_{m} /(1+\mu)$.

$$
w_{x}=\frac{w_{m}}{1+\mu}
$$

The equilibrium conditions for the factor market can be expressed as:

$$
\begin{gathered}
C_{1}^{x}\left(w_{x}, \eta\right) x+(1+\mu) C_{1}^{y}\left(w_{m}, \tau\right) y=L \\
C_{2}^{x}\left(w_{x}, \eta\right) x=T
\end{gathered}
$$

where $C_{j}^{i}(\cdot)$ represents the derivative of the unit cost function of good $i(i \in\{x, y\})$ with respect to $j^{\text {th }}$ argument. $L$ and $T$ are the total population and the land endowment respectively.

The utility function of the representative consumer can be specified as follows:

$$
U\left(c_{x}, c_{y}, z\right)=\frac{1}{\gamma}\left(c_{x}^{\gamma}+c_{y}^{\gamma}\right)^{\frac{1}{\gamma}} z^{-\delta}, 0<\gamma<1, \quad 0<\delta .
$$

The parameter $1 /(1-\gamma)$ is the constant elasticity of substitution. The variable $c_{x}\left(c_{y}\right)$ represents the consumption of good $x(y)$. In the meanwhile, the utility function satisfies the following assumptions: 


$$
\begin{gathered}
U_{x}>0, U_{y}>0, U_{z}>0, U_{x x}<0, U_{y y}<0, \\
U_{x y}=U_{y x}>0, U_{x z}=U_{z x}<0, U_{y z}=U_{z y}<0 .
\end{gathered}
$$

Let $U_{a}$ be the derivative of the utility function with respect to the variable $a$ and $U_{a b}$ be the derivative of $U_{a}$ w.r.t. the variable $b$, where $a, b=x, y, z$. The specifications in utility function imply that the marginal utility of each good is positive and decreasing. The pollution $z$ exerts a negative externality on the level of utility, that is, the marginal utility from pollution is negative. Hence, $\delta$ could represent the degree of disutility of pollution. As customary, the assumption of $U_{x y}=U_{y x}>0$ indicates that the greater consumption of good $x$ will raises the marginal utility of consumption of good $y$, vice versa. Finally, the assumptions, i.e., $U_{x z}=U_{z x}<0$ and $U_{y z}=U_{z y}<0$, state that the larger amount of pollution will lower more environmental quality, and hence reduce the marginal utility of consumption of each good. In this case the relative demand function can be derived as follows:

$$
\frac{c_{x}}{c_{y}}=p^{\frac{1}{1-\gamma}}
$$

Suppose that the government transfers the environmental tax revenue to the consumers in a lump-sum fashion. Hence, we have

$$
c_{x}+p c_{y}=x+p y
$$

The market equilibrium conditions of good $x$ and $y$ are given by $c_{x}=x$ and $c_{y}=y$ because of the assumption of a closed economy. By making use of the Walras' law, only Equation (8) is needed:

$$
c_{y}=y
$$

The general equilibrium satisfies eight simultaneous Equations (1)-(8) and then we can solve eight endogenous variables: $w_{x}, \eta, p, \mu, x, y, c_{x}$, and $c_{y}$, given $w_{m}, \tau, L$, and $T$.

\section{Environmental Policy, Unemployment and Pollution}

In this section, the impact of environmental tax $\tau$ and minimal wage rate $w_{m}$ on unemployment rate will be explored. Without losing generality, we assume that $L$ and $T$ are constant, that is, this paper will consider the effects of $w_{m}$ and $\tau$. Next, following Jones (1965), we can express Equations (1)-(8) in terms of change. Denoting by $(\hat{k}=d k / k)$ the percentage change, totally differentiating Equations (1)-(8), we can obtain the following matrix (see Appendix 1 for the mathematical derivation):

$$
\begin{gathered}
{\left[\begin{array}{ccccc}
\theta_{x L} & \theta_{x T} & 0 & 0 & 0 \\
-\lambda_{x L} \theta_{x T} \sigma^{x} & \lambda_{x L} \theta_{x T} \sigma^{x} & \mu \lambda_{y L} & \lambda_{x L} & (1+\mu) \lambda_{y L} \\
\theta_{x L} \sigma^{x} & -\theta_{x L} \sigma^{x} & 0 & 1 & 0 \\
1 & 0 & \mu /(1+\mu) & 0 & 0 \\
0 & 0 & 0 & 1 & -1
\end{array}\right]\left[\begin{array}{c}
\hat{w}_{x} \\
\hat{\eta} \\
\hat{\mu} \\
\hat{x} \\
\hat{y}
\end{array}\right]} \\
=\left[\begin{array}{c}
0 \\
-(1+\mu) \lambda_{y L} \theta_{y z} \sigma^{y} \\
0 \\
0 \\
\theta_{y z} /(1-\gamma)
\end{array}\right] \hat{\tau}+\left[\begin{array}{c}
0 \\
(1+\mu) \lambda_{y L} \theta_{y z} \sigma^{y} \\
0 \\
1 \\
\theta_{y L} /(1-\gamma)
\end{array}\right] \hat{w}_{m}
\end{gathered}
$$

where the variable $\theta_{i j}(i=x, y ; j=L, T, z)$ represents the share of the $j$ th factor in the value of the $i$ th sector's output, and $\theta_{x L}+\theta_{x T}=\theta_{y L}+\theta_{y z}=1$. The variable $\lambda_{i L}$ denotes the allocation share of labor in sector $i$, i.e., $\lambda_{x L}=L_{x} / L, \lambda_{y L}=L_{m} / L$, and thereby the equilibrium condition for the labor market can be rewrote as $\lambda_{x L}+(1+\mu) \lambda_{y L}=1$. (Note 7) The variable $\sigma^{i}$ is the elasticity of substitution between factors of production in sector $i$. Let $\Delta$ be the determinant of the coefficient matrix in Equation (9), and $\Delta=\left[\mu \sigma^{x}\left(\lambda_{x L} \theta_{x T}+\theta_{x L}\right)\right] /(1+\mu)>0$. Therefore, the results of comparative-static analysis can be derived as follows (see Appendix 2 for the mathematical derivation): 


$$
\begin{aligned}
& \frac{\hat{w}_{x}}{\hat{\tau}}+\frac{\hat{w}_{x}}{\hat{w}_{m}}=\frac{-\gamma \mu \lambda_{y L} \theta_{x T}}{(1-\gamma) \Delta}<0 \\
& \frac{\hat{\eta}}{\hat{\tau}}+\frac{\hat{\eta}}{\hat{w}_{m}}=\frac{\gamma \mu \lambda_{y L} \theta_{x L}}{(1-\gamma) \Delta}>0 \\
& \frac{\hat{\mu}}{\hat{\tau}}+\frac{\hat{\mu}}{\hat{w}_{m}}=\frac{\sigma^{x}\left(\lambda_{x L} \theta_{x T}+\theta_{x L}\right)+(1+\mu) \lambda_{y L} \theta_{x T} /(1-\gamma)}{\Delta}>0 \\
& \frac{\hat{x}}{\hat{\tau}}+\frac{\hat{x}}{\hat{w}_{m}}=\frac{\gamma \mu \lambda_{y L} \theta_{x L} \sigma^{x}}{(1-\gamma) \Delta}>0 \\
& \frac{\hat{y}}{\hat{\tau}}+\frac{\hat{y}}{\hat{w}_{m}}=\frac{-\mu\left\{\lambda_{x L} \sigma^{x} /(1-\gamma)+(1+\mu) \lambda_{y L}\left[\theta_{x T} /(1-\gamma)+\theta_{x L} \sigma^{x}\right]\right\}}{(1+\mu) \Delta}<0
\end{aligned}
$$

Equations (10)-(14) indicate how the increases not only in $\tau$ but also in $w_{m}$ affect five endogenous variables, i.e., $w_{x}, \eta, \mu, x, y$. Next, from Appendix 3 for the mathematical derivation, we get the relationship between the variables (i.e., $\tau$ and $w_{m}$ ) and pollution $z$ as follows:

$$
\frac{\hat{z}}{\hat{\tau}}+\frac{\hat{z}}{\hat{w}_{m}}=\frac{-\mu\left\{\lambda_{x L} \sigma^{x} /(1-\gamma)+(1+\mu) \lambda_{y L}\left[\theta_{x T} /(1-\gamma)+\theta_{x L} \sigma^{x}\right]\right\}}{(1+\mu) \Delta}<0
$$

The economic intuition on Equations (10)-(15) should be stated as follows. If the government increases $\tau$ and $w_{m}$ simultaneously, then the production cost of the urban firm will expand, which will reduce the output of the manufactured good $y$ (Equation (14)). As the output of $y$ decreases, the firm will employ less $z$ and $L_{m}$. That is to say, pollution $z$ unambiguously decreases (Equation (15)). The shrinkage of urban employment $L_{m}$ implies that the urban unemployment rate rises (Equation (12)). The increases not only in $\mu$ but also in $w_{m}$ will reduce the expected urban wage rate $w_{m} /(1+\mu)$, which will in turn lead to a migration from urban area to rural area. (Note 8) That is, in rural sector, the supply of labor will rise, which will raise the output of the agricultural good $x$ (Equation (13)) and reduce $w_{x}$ (Equation (10)). A rise in the output of $x$ leads to an increase in the demand of land and thereby the rental rate of land $\eta$ also rises (Equation (11)). Therefore, as analysis earlier, we can indicate the Proposition 1 as follows:

Proposition 1. When the government raises the environmental tax and the urban minimal wage rate simultaneously, the pollution would decrease and the urban unemployment rate would increase.

\section{Environmental Policy and Welfare}

In this section, we will study the welfare effect of the environmental policy. From the utility function $U\left(c_{x}, c_{y}, z\right)=(1 / \gamma)\left(c_{x}^{\gamma}+c_{y}^{\gamma}\right)^{1 / \gamma} z^{-\delta}$, the indirect utility function $V=(1 / \gamma)\left[1+p^{\gamma /(1-\gamma)}\right]^{1 / \gamma} y z^{-\delta}$ can be obtained. Totally differentiating it yields:

$$
\hat{V}=\frac{1}{1-\gamma} p^{\frac{\gamma}{1-\gamma}}\left(1+p^{\frac{\gamma}{1-\gamma}}\right)^{-1} \hat{p}+\hat{y}-\delta \hat{z}
$$

Therefore, we have (see Appendix 4 for the mathematical derivation):

$$
\frac{\hat{V}}{\hat{\tau}}+\frac{\hat{V}}{\hat{w}_{m}}=\frac{1}{1-\gamma} p^{\frac{\gamma}{1-\gamma}}\left(1+p^{\frac{\gamma}{1-\gamma}}\right)^{-1}+(1-\delta)\left(\frac{\hat{z}}{\hat{\tau}}+\frac{\hat{z}}{\hat{w}_{m}}\right)>0, \text { if } \delta \geq 1
$$

Equation (17) states that, if the degree of disutility of pollution is large enough (i.e., $\delta \geq 1$ ), the policy that the government raises $\tau$ and $w_{m}$ simultaneously will encourage the welfare level. The economic intuition is quite obvious. Proposition 1 indicates that this policy will reduce pollution. Therefore, under the case of the more degree of disutility of pollution, a decrease in pollution will bring the more increase in welfare level. Proposition 2 can be addressed as follows:

Proposition 2. When the government raises the environmental tax and the urban minimal wage rate simultaneously, the welfare level may rise, which depends on the degree of disutility of pollution. 


\section{Concluding Remarks}

In a specific-factor model of a closed HT economy, this paper explore why the government of China imposes on environmental tax and increases the minimum wage simultaneously. We find that the policies of China will lead to a decrease in the pollution and an increase in the urban unemployment rate. In addition, we also prove that the impact on the welfare level will depend on the degree of disutility of pollution. Namely, if the degree of disutility of pollution is large enough, the policies of China government will raise the welfare level. Some policy implications can be drawn from our results. That is, if China government's objective is to increase the welfare level, then it will impose on environmental tax and increase the minimum wage simultaneously.

\section{Acknowledgements}

I am grateful to the Editor and the anonymous referees for useful comments on the earlier version of this paper. Any remaining errors are my responsibility.

\section{References}

Beghin, J., Roland-Holst, D., \& Van der Mensbrugghe, D. (2002). Trade and the environment in general equilibrium: Evidence from developing economies. Dordrecht: Kluwer Academic. http://dx.doi.org/10.1007/0-306-47672-X

Beladi, H., \& Chao, C. C. (2006). Environmental policy, comparative advantage, and welfare for a developing country. Environment and Development Economics, 11, 559-568. http://dx.doi.org/10.1017/S1355770X06003123

Chao, C. C., Kerkvliet, J. R., \& Yu, E. S. H. (2000). Environmental preservation, sectoral unemployment, and trade in resources. Review of Development Economics, 4, 39-50. http://dx.doi.org/10.1111/1467-9361.00077

Copeland, B. R., \& Taylor, S. M. (1994). North-south trade and the environment. Quarterly Journal of Economics, 109, 755-787. http://dx.doi.org/10.2307/2118421

Daitoh, I. (2003). Environmental protection and urban unemployment: Environmental policy reform in a polluted dualistic economy. Review of Development Economics, 7, 496-509. http://dx.doi.org/10.1111/1467-9361.00205

Daitoh, I. (2008). Environmental protection and trade liberalization in a small open dual economy. Review of Development Economics, 12, 728-736.

Daitoh, I. (2012). Environmental sustainability and poverty reduction in a small open dualistic economy: The case of wage subsidy policies and industrial pollution under sector-specific capital. In M. C. Joanne (Ed.), Developing economies: Innovation, investment and sustainability (pp. 157-174). New York: Nova Science Publishers, Inc.

Daitoh, I., \& Omote, M. (2011). The optimal environmental tax and urban unemployment in an open economy. Review of Development Economics, 15, 168-179. http://dx.doi.org/10.1111/j.1467-9361.2010.00600.x

Dean, J. M., \& Gangopadhyay, S. (1997). Export bans, environmental protection, and unemployment. Review of Development Economics, 1, 324-336. http://dx.doi.org/10.1111/1467-9361.00022

Harris, J. R., \& Todaro, M. P. (1970). Migration, unemployment and development: A two-sector analysis. American Economic Review, 60, 126-142.

Jones, R. W. (1965). The structure of simple general equilibrium models. Journal of Political Economy, 73, 557572. http://dx.doi.org/10.1086/259084

McGuire, M. C. (1982). Regulation, factor rewards, and international trade. Journal of Public Economics, 17, 335-354. http://dx.doi.org/10.1016/0047-2727(82)90069-X

Pethig, R. (1976). Pollution, welfare, and environmental policy in the theory of comparative advantage. Journal of Environmental Economics and Management, 2, 160-169. http://dx.doi.org/10.1016/0095-0696(76)90031-0

Rapanos, V. T. (2007). Environmental taxation in a dualistic economy. Environment and Development Economics, 12, 73-89. http://dx.doi.org/10.1017/S1355770X06003391

Tsakiris, N., Hatzipanayotou, P., \& Michael, M. S. (2008). Pollution, capital mobility and tax policies with

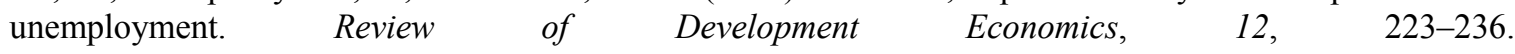
http://dx.doi.org/10.1111/j.1467-9361.2007.00383.x 


\section{Notes}

Note 1. The same notion, please see Beghin et al. (2002), Daitoh (2003) and Daitoh and Omote (2011).

Note 2. Please also see "The China carbon price", The Economist, Aug. 10, 2009, http://www.economist.com/node/20024590.

Note 3. Please see CommonWealth, Mar. 24, 2011, http://www.cw.com.tw/article/article.action?id=5006563.

Note 4. Dean and Gangopadhyay (1997) set up a three-sector model assuming that the production of intermediates causes environmental damage to explore the impact of an export ban on intermediate goods. They argue that the export ban increases urban unemployment in the short run, but decreases it in the long run. Chao et al. (2000) explore the relationship between environmental protection and urban unemployment. They prove that increasing environmental protection leads to an increase in urban unemployment in a closed economy, but it does not result in additional urban unemployment in a small open economy. In the standard model of a closed HT economy, Daitoh (2003) shows that the effects of the environmental policy on the manufacturing employment depend on the price elasticity of demand for manufactured goods. Daitoh (2008) extends Daitoh's (2003) model to an open economy and then proves that the former condition depends on the substitute or complementary relations among factors of production. Daitoh (2012) analyzes effects of wage subsidy policies on urban pollution in a dualistic economy. Beladi and Chao (2006) derive the optimal environmental tax rate under urban unemployment. Rapanos (2007) finds that the environmental tax may increase or decrease urban unemployment in the short run, while it decreases unemployment in the long run. Tsakiris et al. (2008) show that the optimal environmental tax is lower than the marginal damage of pollution. Daitoh and Omote (2011) find that a rise in the pollution tax rate would lead to an increase in the urban unemployment. In sum, the impact of environmental protection on urban unemployment and welfare is inconclusive.

Note 5. Please see Harris and Todaro (1970).

Note 6. The similar specification of treating pollution as a factor of production can also be seen in Pethig (1976), McGuire (1982), Copeland and Taylor (1994), Daitoh (2003; 2008) and Daitoh and Omote (2011). If pollution $z$ is a factor of production, then environmental tax $\tau$ could be interpreted as its price.

Note 7. Using Equation (4), we have $L_{x}+(1+\mu) L_{m}=L$. Next, dividing each side of the equality by $L$ can obtain $\left(L_{x} / L\right)+(1+\mu)\left(L_{m} / L\right)=1$, that is, the result of $\lambda_{x L}+(1+\mu) \lambda_{y L}=1$ can be found.

Note 8. Harris and Todaro (1970) argue that the impact of the minimal wage rate $w_{m}$ on the expected urban wage rate is ambiguous. They address that a rise in $w_{m}$ would lead to a migration from rural sector to urban sector and hence the urban unemployment rate rises, but the expected urban wage rate is inconclusive. Similarly, Daitoh (2003) claims that if the pollution tax $\tau$ rises then $L_{x}$ may increase or decrease. However, in this paper, the case that $\tau$ and $w_{m}$ are raised simultaneously is discussed, which means that the prices of all production factors rise in the urban firm, and hence the output of the manufactured goods in this case is less than in Harris and Todaro (1970). To understand it more clearly, one should pay attention to the fact that the less output of the manufactured good would lead to the more shrinkage of urban employment, that is, the more urban unemployment rate would happen. If the effects of the urban unemployment are large enough then the result of a migration from urban area to rural area would be obtained.

\section{Appendix}

Appendix 1

Totally differentiating Equations (1)-(5), we can find:

$$
\begin{gathered}
\theta_{x L} \hat{w}_{x}+\theta_{x T} \hat{\eta}=0 \\
\theta_{y L} \hat{w}_{m}+\theta_{y z} \hat{\tau}=\hat{p} \\
\hat{w}_{x}=\hat{w}_{m}-\frac{\mu}{1+\mu} \hat{\mu} \\
-\lambda_{x L} \theta_{x T} \sigma^{x} \hat{w}_{x}+\lambda_{x L} \theta_{x T} \sigma^{x} \hat{\eta}+\lambda_{x L} \hat{x}+(1+\mu) \lambda_{y L} \hat{y}+\mu \lambda_{y L} \hat{\mu}-(1+\mu) \lambda_{y L} \theta_{y z} \sigma^{y} \hat{w}_{m} \\
+(1+\mu) \lambda_{y L} \theta_{y z} \sigma^{y} \hat{\tau}=\hat{L}=0 \\
\theta_{x L} \sigma^{x} \hat{w}_{x}-\theta_{x L} \sigma^{x} \hat{\eta}+\hat{x}=\hat{T}=0
\end{gathered}
$$


Substituting Equations (7) and (8) into Equation (6) and then totally differentiating it can obtain:

$$
\hat{x}-\hat{y}=\frac{1}{1-\gamma} \hat{p}
$$

By substituting Equation (A.2) into (A.6), we can get:

$$
\hat{x}-\hat{y}=\frac{\theta_{y L}}{1-\gamma} \hat{w}_{m}+\frac{\theta_{y z}}{1-\gamma} \hat{\tau}
$$

Finally, Equation (9) can be obtained by making use of Equations (A.1), (A.4), (A.5), (A.3), and (A.7).

Appendix 2

By using the Cramer's rule, from Equation (9), we can derive the results of comparative-static analysis as follows:

$$
\begin{aligned}
& \frac{\hat{w}_{x}}{\hat{\tau}}=\frac{\mu \lambda_{y L} \theta_{x T} \theta_{y z}\left[\sigma^{y}-1 /(1-\gamma)\right]}{\Delta} \\
& \frac{\hat{\eta}}{\hat{\tau}}=\frac{-\mu \lambda_{y L} \theta_{x L} \theta_{y z}\left[\sigma^{y}-1 /(1-\gamma)\right]}{\Delta} \\
& \frac{\hat{\mu}}{\hat{\tau}}=\frac{-(1+\mu) \lambda_{y L} \theta_{x T} \theta_{y z}\left[\sigma^{y}-1 /(1-\gamma)\right]}{\Delta} \\
& \frac{\hat{x}}{\hat{\tau}}=\frac{-\mu \lambda_{y L} \theta_{x L} \theta_{y z} \sigma^{x}\left[\sigma^{y}-1 /(1-\gamma)\right]}{\Delta} \\
& \frac{\hat{y}}{\hat{\tau}}=\frac{-\mu \theta_{y z}\left[\lambda_{x L} \sigma^{x} /(1-\gamma)+(1+\mu) \lambda_{y L} \theta_{x L} \sigma^{x} \sigma^{y}+(1+\mu) \lambda_{y L} \theta_{x T} /(1-\gamma)\right]}{(1+\mu) \Delta}<0, \\
& \frac{\hat{w}_{x}}{\hat{w}_{m}}=\frac{\mu \lambda_{y L} \theta_{x T}\left[1-\theta_{y z} \sigma^{y}-\theta_{y L} /(1-\gamma)\right]}{\Delta} \\
& \frac{\hat{\eta}}{\hat{w}_{m}}=\frac{-\mu \lambda_{y L} \theta_{x L}\left[1-\theta_{y z} \sigma^{y}-\theta_{y L} /(1-\gamma)\right]}{\Delta} \\
& \frac{\hat{\mu}}{\hat{w}_{m}}=\frac{\sigma^{x}\left(\lambda_{x L} \theta_{x T}+\theta_{x L}\right)+(1+\mu) \lambda_{y L} \theta_{x T}\left[\theta_{y z} \sigma^{y}+\theta_{y L} /(1-\gamma)\right]}{\Delta}>0 \\
& \frac{\hat{x}}{\hat{w}_{m}}=\frac{-\mu \lambda_{y L} \theta_{x L} \sigma^{x}\left[1-\theta_{y z} \sigma^{y}-\theta_{y L} /(1-\gamma)\right]}{\Delta} \\
& \frac{\hat{y}}{\hat{w}_{m}}=\frac{\mu}{(1+\mu) \Delta}\left\{-(1+\mu) \lambda_{y L} \theta_{x L} \sigma^{x}\left[1-\theta_{y z} \sigma^{y}-\theta_{y L} /(1-\gamma)\right]+\right. \\
& \left.\left[\theta_{y L} /(1-\gamma)\right]\left\{\sigma^{x}\left[(1+\mu) \lambda_{y L} \theta_{x T}-1\right]-(1+\mu) \lambda_{y L} \theta_{x T}\right\}\right\} .
\end{aligned}
$$

Appendix 3

Since $z=C_{2}^{y}\left(w_{m}, \tau\right) y$, totally differentiating this we get $\hat{z}=\theta_{y L} \sigma^{y} \hat{w}_{m}-\theta_{y L} \sigma^{y} \hat{\tau}+\hat{y}$. Therefore, the impact of environmental tax on pollution can be derived as follows:

$$
\frac{\hat{z}}{\hat{\tau}}=-\theta_{y L} \sigma^{y}+\frac{\hat{y}}{\hat{\tau}}<0
$$

Similarly, we can find the effect of minimal wage rate on pollution as follows: 


$$
\frac{\hat{z}}{\hat{w}_{m}}=\theta_{y L} \sigma^{y}+\frac{\hat{y}}{\hat{w}_{m}}
$$

Appendix 4

From Equation (16), we have

$$
\frac{\hat{V}}{\hat{\tau}}=\frac{1}{1-\gamma} p^{\frac{\gamma}{1-\gamma}}\left(1+p^{\frac{\gamma}{1-\gamma}}\right)^{-1} \frac{\hat{p}}{\hat{\tau}}+\frac{\hat{y}}{\hat{\tau}}-\delta \frac{\hat{z}}{\hat{\tau}}
$$

and

$$
\frac{\hat{V}}{\hat{w}_{m}}=\frac{1}{1-\gamma} p^{\frac{\gamma}{1-\gamma}}\left(1+p^{\frac{\gamma}{1-\gamma}}\right)^{-1} \frac{\hat{p}}{\hat{w}_{m}}+\frac{\hat{y}}{\hat{w}_{m}}-\delta \frac{\hat{z}}{\hat{w}_{m}}
$$

Combining Equations (A.20) with (A.21) can obtain Equation (A.22) which is equal to Equation (17) as follows:

$$
\begin{gathered}
\frac{\hat{V}}{\hat{\tau}}+\frac{\hat{V}}{\hat{w}_{m}}=\frac{1}{1-\gamma} p^{\frac{\gamma}{1-\gamma}}\left(1+p^{\frac{\gamma}{1-\gamma}}\right)^{-1}\left(\frac{\hat{p}}{\hat{\tau}}+\frac{\hat{p}}{\hat{w}_{m}}\right)+\left(\frac{\hat{y}}{\hat{\tau}}+\frac{\hat{y}}{\hat{w}_{m}}\right)-\delta\left(\frac{\hat{z}}{\hat{\tau}}+\frac{\hat{z}}{\hat{w}_{m}}\right) \\
=\frac{1}{1-\gamma} p^{\frac{\gamma}{1-\gamma}}\left(1+p^{\frac{\gamma}{1-\gamma}}\right)^{-1}+(1-\delta)\left(\frac{\hat{z}}{\hat{\tau}}+\frac{\hat{z}}{\hat{w}_{m}}\right)
\end{gathered}
$$

\section{Copyrights}

Copyright for this article is retained by the author(s), with first publication rights granted to the journal.

This is an open-access article distributed under the terms and conditions of the Creative Commons Attribution license (http://creativecommons.org/licenses/by/3.0/). 